\title{
Distinguishing Phosphorylation and Sulfation in Carbohydrates and Glycoproteins Using Ion-Pairing and Mass Spectrometry
}

\author{
Ying Zhang, Hui Jiang, Eden P. Go, and Heather Desaire \\ Department of Chemistry, University of Kansas, Lawrence, Kansas, USA
}

\begin{abstract}
Phosphorylation and sulfation are important modifications affecting the biological properties of carbohydrates, proteins, and glycoproteins. Identification of these two functional groups facilitates the understanding of the structure/function relationship in various species. Mass spectrometry is one of the methods used to detect the presence of these two modifications in complex biological mixtures. However, phosphorylated and sulfated structures are isobaric; thus, differentiation between them in routinely used mass spectrometers is problematic. Herein, we demonstrate that these two groups can be discriminated by using ion-pairing in conjunction with MS/MS experiments. The characteristic product ions are used to successfully identify the phosphorylation and sulfation present in mono-, disaccharides, and the highly sulfated glycoprotein, ovine luteinizing hormone. This method is a robust approach to differentiate the two isobaric functional groups. (J Am Soc Mass Spectrom 2006, 17, 1282-1288) (C) 2006 American Society for Mass Spectrometry
\end{abstract}

$\mathrm{P}$ hosphorylation and sulfation are significant biological modifications that affect the functions of numerous species, carbohydrates [1], proteins [2-5], and glycoproteins [6-15]. For example, tyrosine phosphorylation of proteins in the cytoplasm plays an essential role in intracellular signaling transduction [2,3], cell cycle control [4], transcription of gene expression [4], cytoskeletal regulation, and apoptosis $[3,4]$. Tyrosine sulfation results in different functions, including modulation of intracellular protein transportation, regulation of proteolytic process of proteins, and the alteration of biological activity of proteins $[3,5-7]$. Phosphorylation and sulfation present on carbohydrate moieties of glycoproteins also causes different biological responses. Changes in sulfation on the glycoproteins have been related to various diseases, such as diabetes, arthritis, Alzheimer's disease, and cystic fibrosis etc. [8]. In addition, alterations in phosphorylation of carbohydrate moieties on glycoproteins are also linked to diverse types of diseases, including the neuronal ceroid lipofuscinoses (NCLs), which are hereditary neurodegenerative diseases affecting human beings [9]. Moreover, phosphorylated glycoproteins, such as mannose-6-phosphorylated glycoprotein, can be used as prognostic markers for breast cancer, ovarian cancer, and prostate cancer, etc. [10]. Since phosphorylation and sulfation have been associated with significantly different biological functions, developing efficient and sensitive methods to discriminate these structures is essential for understanding their roles in protein functions and in diseases.

Published online July 3, 2006

Address reprint requests to Dr. H. Desaire, Department of Chemistry, University of Kansas, 1251 Wescoe Hall Drive, Lawrence, KS 66045, USA. E-mail: hdesaire@ku.edu
Traditionally, phosphorylation and sulfation of carbohydrates or proteins are detected by radiolabeling using ${ }^{32} \mathrm{P}$ or ${ }^{35} \mathrm{~S}[2,6,7,14-17]$ isotopes, separation using gel-filtration chromatography [11-13], high-performance liquid chromatography (HPLC) [11-13, 16, 17], twodimensional thin-layer chromatography (TLC) $[7,16]$, and the detection of these isotopes in scintillation counting or autoradiography [16]. While radiolabeling is effective at identifying the presence of phosphorylation or sulfation, the detection step must be followed by additional characterization steps, since determining the presence of the radioisotopes does not provide any information about the structure of the compound that was labeled.

In recent years, mass spectrometry has become a powerful analytical tool in the identification and characterization of biological molecules and their posttranslational modifications. It is highly sensitive and selective, and provides rapid means to analyze samples, obviating the need for radiolabeling [16, 18]. A MS-based method to discriminate between phosphorylation and sulfation involves reacting suspected phosphorylated compounds with alkaline phosphatase [16]. MS analysis is applied before and after treatment with the enzyme to detect the loss of phosphate in the products [16]. Although this type of enzymatic assay is effective in differentiation between phosphorylation and sulfation, its application is limited when analyzing complex mixtures, which imparts a significant barrier in many biological applications, especially when the compounds are phosphorylated or sulfated at low levels [16].

High-resolution MS methods, such as Fourier transform ion cyclotron resonance mass spectrometry (FTICRMS), have been used to distinguish between phosphorylated and sulfated compounds based on their slight 
difference in mass [19] or by their ability to react differently in ion-molecule reactions [20]. Other MS methods, such as matrix-assisted laser desorption/ionization (MALDI) [21] and electrospray ionization (ESI) [22], have been recently reported to distinguish phosphorylation and sulfation. In MALDI analysis, sulfated and phosphorylated products ionize somewhat differently, and these differences could potentially be used to distinguish phosphate and sulfate in an unknown compound [21]. In ESI analysis, ion-pairing reagents have been utilized to complex with singly phosphorylated or sulfated monosaccharides [22]. The isobaric ion-pairing complexes can be differentiated in MS/MS experiments because of their different chemical reactivities. The fundamental study indicates that this approach is only functional group dependent [22] and, thus, it could potentially be effective in discriminating the phosphorylation and sulfation in larger biologically relevant molecules. If the ion-pairing method, which distinguishes phosphorylated and sulfated monosaccharides, could be adapted to larger macromolecules, such as oligosaccharides and glycoproteins, this approach would afford a unique and effective way to distinguish phosphorylation from sulfation. Using ionpairing to discriminate these groups is advantageous to other methods because this approach provides significant advantages that are not related to phosphate/sulfate discrimination: The ion-pairing method has already been demonstrated to enhance the signal of sulfated glycopeptides [23], and to facilitate structural elucidation of the glycan moiety present in glycoproteins [24]. Thus, sulfate/ phosphate determination could be accomplished in a synergistic analysis that also enhances sensitivity and provides additional structural information about glycoproteins.

In the work presented here, the ion-pairing approach described previously to discriminate monosaccharides [22] is utilized to identify the phosphorylation and sulfation present in disaccharides and glycopeptides generated from a proteolytic digest of ovine luteinizing hormone $\alpha$-subunit (oLH $\alpha$ ). Difference in chemical reactivities between these two acidic functional groups observed in the monosaccharides [22] can be preserved when this approach is applied to complex biomolecules. This is the first study to use the ion-pairing technique in conjunction with MS/MS to differentiate phosphorylation and sulfation on disaccharides and glycopeptides. In addition to discriminating the isobaric compounds, this approach can also simultaneously enhance the mass spectral signal of acidic groups in complex mixtures by enhancing the ionization efficiency of these functional groups [23].

\section{Materials and Methods}

\section{Materials}

Phosphorylated or sulfated monosaccharides, disaccharides, and the peptide Lys-Lys-Lys were purchased from Sigma-Aldrich (St. Louis, MO) and were used without further purification. Glycopeptides from ovine luteinizing hormone, $\alpha$-subunit, $(\mathrm{oLH} \alpha)$ were produced in the laboratory of Dr. George Bousfield, Wichita State University, as described elsewhere [23, 25].

\section{Ion-Pairing Experiments}

Ion-pair complexes were formed by combining equal volumes of basic peptide, Lys-Lys-Lys, (henceforth referred to as K3), with solutions of monosaccharides or disaccharides. Phosphorylated or sulfated carbohydrates and $\mathrm{K} 3$ were dissolved in a minimal amount of HPLC grade water, respectively. Carbohydrate solutions were further diluted with a methanol:water $\left(\mathrm{MeOH}: \mathrm{H}_{2} \mathrm{O}\right)$ mixture (1:1) containing $0.5 \%$ acetic acid, to the final concentration of $1 \mathrm{mM}$. The peptide, $\mathrm{K} 3$, was diluted to $2 \mathrm{mM}$. Ion-pair complexes were generated by mixing $5 \mu \mathrm{L}$ of carbohydrate solution with $5 \mu \mathrm{L}$ of peptide solution, vortexing and injecting $2 \mu \mathrm{L}$ of this mixture into the mass spectrometer. The ion-pair complexes of glycopeptides with $\mathrm{K} 3$ were formed by dissolving $1 \mu \mathrm{g}$ of the glycopeptide mixture in $3 \mu \mathrm{L}$ of a $1.25 \mathrm{mM}$ solution of $\mathrm{K} 3$. One to three $\mu \mathrm{L}$ of this sample were injected into the mass spectrometer.

\section{Mass Spectrometry}

All the ion-pair complexes were monitored on a Thermo Finnigan LCQ Advantage quadrupole ion trap mass spectrometer (Thermo, San Jose, CA). A surveyor MS-pump (Thermo) was used to deliver the mobile phase $\mathrm{MeOH}$ : $\mathrm{H}_{2} \mathrm{O}$ mixture (1:1) containing $0.5 \%$ acetic acid at a flow rate of $20 \mu \mathrm{L} / \mathrm{min}$. Ion-pair complexes were directly introduced to the mass spectrometer, and all the mass spectra were acquired in the positive ion mode. Electrospray ionization was achieved by using a spray voltage of 4.0 KV. Nitrogen was used as nebulizing gas, at a pressure of 20 psi. The capillary temperature was maintained at $230^{\circ} \mathrm{C}$.

Collision-induced dissociation (CID) was performed to obtain structural information about the ion-pairs. Specifically, for the phosphorylated or sulfated monoand disaccharide ion-pair complexes, doubly charged precursor ions were activated for $30 \mathrm{~ms}$ with $20 \%$ normalized collision energy. $\mathrm{A} \mathrm{q}_{\mathrm{z}}$ value of 0.25 and an isolation width of $3 \mathrm{Da}$ were used. For the ion-pairing complexes containing glycopeptides released from $\mathrm{oLH} \alpha$ and the peptide K3, doubly charged precursor ions were activated with $19 \%$ normalized collision energy. All the data were acquired and analyzed using Xcalibur 1.3 software (Thermo, San Jose, CA).

\section{Results and Discussion}

Ion pairing complexes readily form when the ion-pairing reagent is mixed with sulfated/phosphorylated carbohydrates. An example of the resulting MS data is shown in Figure 1. Recently, we demonstrated that phosphorylated and sulfated monosaccharides can be differentiated based on the different characteristic product ions of singly or 


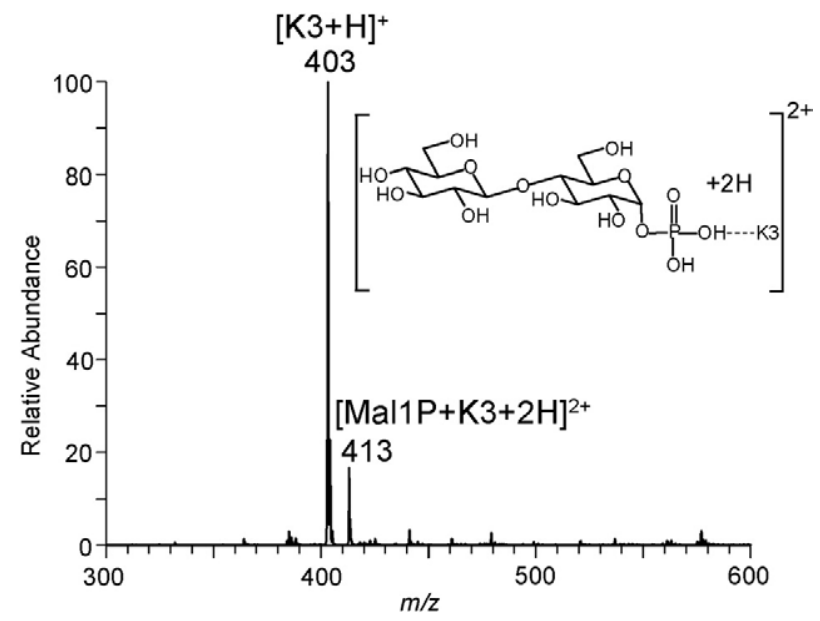

Figure 1. (+) ESI-MS data for the phosphorylated disaccharide maltose-1-phosphate (Mal1P) after the addition of the ion-pairing reagent Lys-Lys-Lys (K3). The spectrum shows two ions, [K3 + $\mathrm{H}]^{+}$and $[\mathrm{Mal1P}+\mathrm{K} 3+2 \mathrm{H}]^{2+}$.

doubly charged ion-pairing complexes; see Table 1 [22]. For doubly charged complexes, sulfated monosaccharides undergo sulfur-oxygen bond cleavage while phosphorylated monosaccharides dissociate by disrupting the noncovalent interaction between the carbohydrate and the ion-pairing reagent. Since HexNAc contain an amide group in their structure, the ligand dissociation can also be observed for the doubly charged, sulfated ion-pairs. For singly charged complexes, sulfated complexes undergo covalent cleavage, resulting in the losses of water, while the phosphorylated complexes undergo cleavage of noncovalent interactions, resulting in the presence of the singly charged peptide ion $[\mathrm{K} 3+\mathrm{H}]^{+}[22]$. The differences in the fragmentation pattern of these two modifications are induced by different chemical reactivities resulting from the slightly different $\mathrm{pKa}$ values of these two acidic groups. Since the ion-pairing MS/MS method has been demonstrated to be functional group specific, it should be useful in identifying phosphorylation and sulfation in more complex carbohydrates and glycopeptides.

\section{MS/MS of Doubly Charged Monosaccharide:K3 Ion Pairs}

Owing to the successful application of ion-pairing technique in discriminating monophosphorylated and sul-

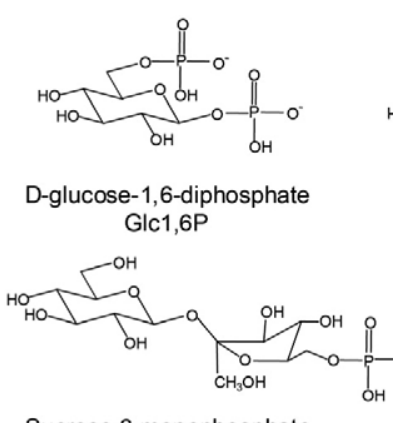

Sucrose 6-monophosphate Suc $6 \mathrm{P}$

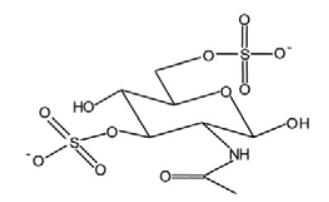

$\mathrm{N}$-acetyl-glucosamine 3,6-disulfate GIcNAc3,6S

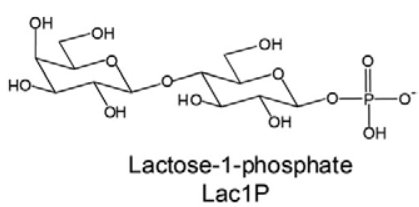

Lac1P

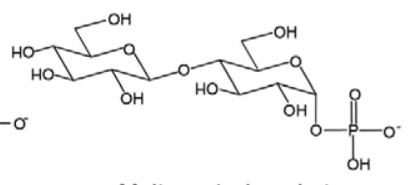

Maltose-1-phosphate

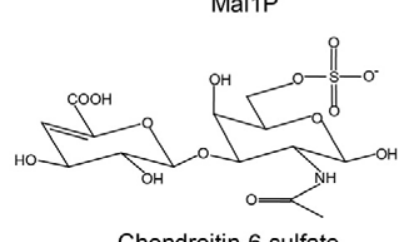

Chondroitin-6-sulfate $\Delta$ di-6S

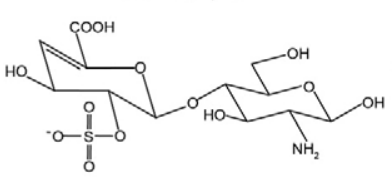

Heparin Dissaccharide IIIH $\mathrm{IIH}$

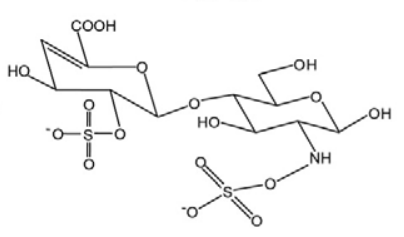

Heparin Dissaccharide IIIS

IIIS
Figure 2. Structures of phosphorylated and sulfated carbohydrates used for the ion-pairing experiments.

fated carbohydrates, we extend its utility in discriminating biologically relevant molecules. To this end, several diphosphorylated and disulfated monosaccharides, along with sulfated and phosphorylated disaccharides, were selected (Figure 2). An initial set of experiments was performed to establish whether the characteristic product ions in Table 1 are preserved for diphosphorylated and disulfated monosaccharides, D-glucose-1,6-diphosphate (Glc1,6P) and N-acetylglucosamine 3,6-disulfate (GlcNAc 3,6S). MS/MS data (Figure 3) show that the mass of doubly charged precursor ions at $\mathrm{m} / \mathrm{z} 372$ and 393 are consistent with the expected mass of the ion pairs [Glc1,6P $+\mathrm{K} 3+2 \mathrm{H}]^{2+}$ and $[\mathrm{GlcNAc} 3,6 \mathrm{~S}+\mathrm{K} 3+2 \mathrm{H}]^{2+}$, respectively. Two complementary ions [Glc1,6P $+\mathrm{H}]^{+}$at $m / z 341$ and [K3 $+\mathrm{H}]^{+}$at $m / z 403$ correspond to the dissociation of the

Table 1. Characteristic product ions used to differentiate phosphate and sulfate in monosaccharides

\begin{tabular}{|c|c|c|c|c|c|}
\hline \multirow[b]{3}{*}{$\begin{array}{l}\text { Observed reaction } \\
\text { pathways }\end{array}$} & \multicolumn{4}{|c|}{ Doubly charged ion pairs } & \multirow{3}{*}{$\begin{array}{l}\text { Singly charged ion pairs } \\
{[\text { Carbohydrates }+\mathrm{K} 3+\mathrm{H}]^{+}} \\
\text {Ligand dissociation }\end{array}$} \\
\hline & \multicolumn{2}{|c|}{$[\text { Hexose }+\mathrm{K} 3+2 \mathrm{H}]^{2+}$} & \multicolumn{2}{|c|}{$[\mathrm{HexNAc}+\mathrm{K} 3+2 \mathrm{H}]^{2+}$} & \\
\hline & $\begin{array}{l}\text { Oxygen-sulfur } \\
\text { (or oxygen- } \\
\text { phosphorus) } \\
\text { bond cleavage }\end{array}$ & $\begin{array}{l}\text { Ligand } \\
\text { dissociation }\end{array}$ & $\begin{array}{l}\text { Oxygen-sulfur } \\
\text { (or oxygen- } \\
\text { phosphorous) } \\
\text { bond cleavage }\end{array}$ & $\begin{array}{l}\text { Ligand } \\
\text { dissociation }\end{array}$ & \\
\hline Sulfate & yes & no & yes & yes* & no \\
\hline Phosphate & no & yes & no & yes & yes \\
\hline
\end{tabular}

*: Ligand dissociation in sulfated HexNAcs is caused by the amine group on the carbohydrate. 

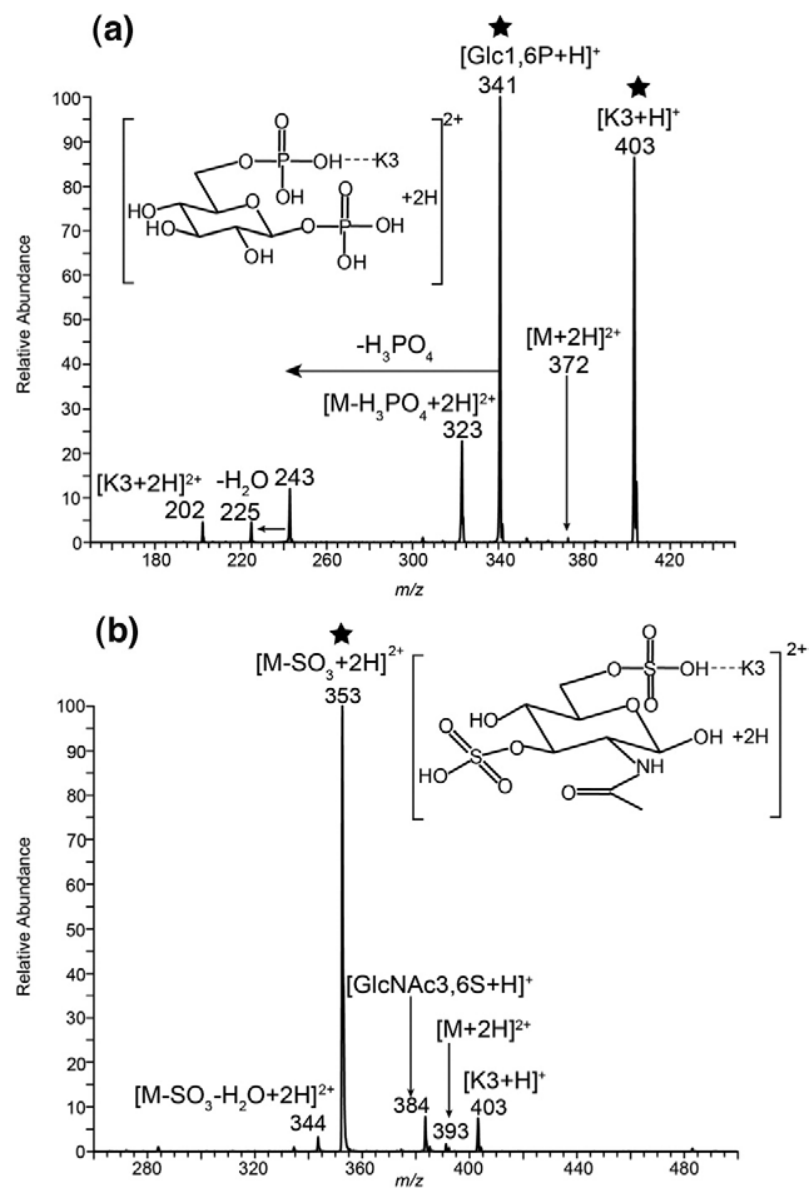

Figure 3. (+) ESI-MS/MS data for the doubly charged diphosphorylated or disulfated ion pairs with Lys-Lys-Lys (K3): (a) diphosphorylated monosaccharide complex [Glc1,6P $+\mathrm{K} 3+$ $2 \mathrm{H}]^{2+}$, (b) disulfated monosaccharide complex [GlcNAc3,6S + K3 $+2 \mathrm{H}]^{2+}$. In (a), the precursor ion underwent the ligand dissociation upon CID, while (b) shows characteristic ions resulting from oxygen-sulfur bond cleavage. Characteristic ions are marked with a star.

noncovalent interaction between the carbohydrate and the ion-pairing reagent, K3, in Figure 3a. This ligand dissociation is much more abundant for the phosphorylated complex compared with the sulfated complex, because the phosphate-peptide interaction is weaker than the sulfate-peptide interaction [22]. As expected, ligand dissociation is the most favorable fragmentation pathway in phosphorylated ion pairs.

In Figure $3 \mathbf{b}$, the ion at $m / z 353$, which originates from oxygen - sulfur bond cleavage, is observed as the base peak. Since sulfated monosaccharide:peptide complex is more stable than the phosphorylated ion pairs, the covalent bond dissociates preferentially compared with the noncovalent bonds [26]. In addition, two small product ions at $\mathrm{m} / \mathrm{z} 384$ and 403 correspond to the ligand dissociation from the precursor ion. This is due to the presence of the amide group on the sulfated HexNAc (Table 1), facilitating the proton transfer [22]. As a result, the observed abundant characteristic product ions in Figure 3 indicate that ion-pairing is useful in discriminating diphosphorylated and disulfated monosaccharides.

\section{MS/MS of Doubly Charged Disaccharide:K3 Ion Pairs}

Aside from the monosaccharides, the ion-pairing approach is also applied to the structurally more complex disaccharides that are phosphorylated or sulfated. Three phosphorylated disaccharides, maltose-1-phosphate (Mal1P), lactose-1phosphate (Lac1P), sucrose 6-monophosphate (Suc6P), and three sulfated disaccharides, heparin disaccharide $\mathrm{IIIH}$ (IIIH), heparin disaccharide IIIS (IIIS), chondroitin-6sulfate ( $\Delta$ di-6S) (Figure 2$)$, were complexed with the peptide, K3. MS/MS data of two isomeric phosphorylated disaccharide:peptide ion-pairs (maltose-1-phosphate:K3 and lactose-1-phosphate:K3) in Figure 4 demonstrate that the fragmentation pattern of these two structures is similar when the complexes are subjected to CID. In both spectra (Figure $4 \mathrm{a}$ and $\mathrm{b}$ ), the precursor ions at $\mathrm{m} / \mathrm{z} 413$ correspond to the doubly charged ions, [Mal1P $+\mathrm{K} 3+2 \mathrm{H}]^{2+}$ and $[\mathrm{Lac} 1 \mathrm{P}+\mathrm{K} 3+2 \mathrm{H}]^{2+}$, respectively. Two complementary ions, $[\mathrm{Mal1P}+\mathrm{H}]^{+}$(Figure 4a) or [Lac1P $\left.+\mathrm{H}\right]^{+}$(Figure $4 b$ ) at $m / z 423$ and the peptide $\mathrm{K} 3$ ion at $m / z$ 403, originate from ligand dissociation. Both characteristic ions are consistent with the expected fragmentation trends described in Table 1. Two interesting ions at $\mathrm{m} / \mathrm{z} 364$ and 325 in Figure $4 \mathrm{a}$ and $\mathrm{b}$ correspond to the loss of $\mathrm{H}_{3} \mathrm{PO}_{4}$. The loss of $\mathrm{H}_{3} \mathrm{PO}_{4}$ only occurs when the carbohydrates are phosphorylated at the anomeric carbon [22]. Thus, the loss of $\mathrm{H}_{3} \mathrm{PO}_{4}(98 \mathrm{Da})$ can be used to identify the position of the phosphate on the structure [22].

Figure 4c shows the MS/MS data of the doubly charged phosphorylated complex [Suc6P $+\mathrm{K} 3+$ $2 \mathrm{H}]^{2+}$. In this spectrum, the parent ion at $\mathrm{m} / \mathrm{z} 413$ dissociates into two singly charged characteristic ions, the peptide ion $(\mathrm{K} 3, \mathrm{~m} / \mathrm{z} 403)$ and the protonated disaccharide ion $(m / z 423)$. All the other product ions are related to the dissociation of the glycosidic bonds and the loss of water. In summary, all the phosphorylated disaccharide complexes dissociated according to the fragmentation trends that were established based on the dissociations of phosphorylated monosaccharide/ionpair complexes.

The fragmentation of the sulfated disaccharide:peptide ion-pairs was also investigated, employing the same collisional dissociation (Figure 5). For the doubly charged complex $[\mathrm{IIIH}+\mathrm{K} 3+2 \mathrm{H}]^{2+}$ in Figure $5 \mathrm{a}$, the singly charged ion at $\mathrm{m} / \mathrm{z} 338$ corresponds to the characteristic ion resulting from oxygen - sulfur bond cleavage. Due to the presence of the amine group in the structure of IIIH, two complementary ions, the singly charged peptide ion at $\mathrm{m} / \mathrm{z} 403$ and the protonated disaccharide ion at $m / z 418$, were observed (Table 1 ).

Figure $5 \mathrm{~b}$ shows the MS/MS data of the doubly charged ion-pair complex, [IIIS $+\mathrm{K} 3+2 \mathrm{H}]^{2+}$, at $\mathrm{m} / \mathrm{z}$ 450.4. Four characteristic ions at $m / z$ 338.0, 417.8, 483.0, and the base peak ion at $\mathrm{m} / \mathrm{z} 410.6$ originate from 

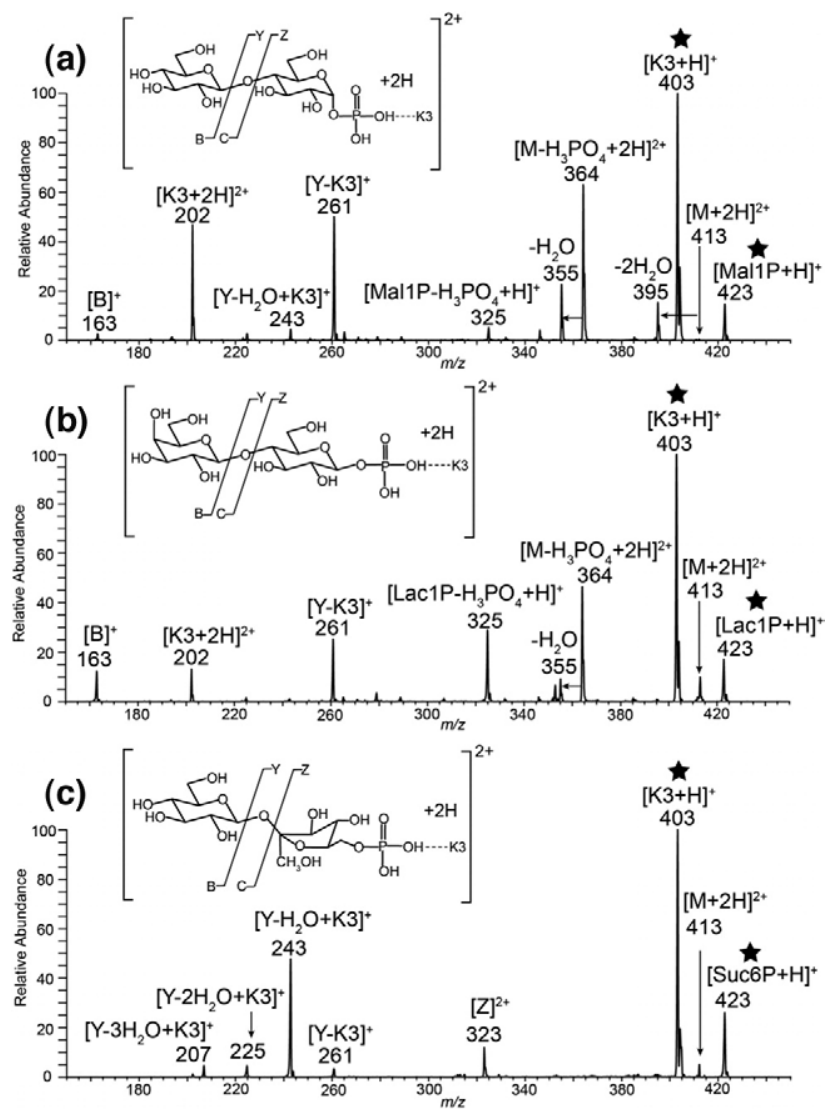

Figure 4. (+) ESI-MS/MS data for the doubly charged phosphorylated ion pairs with Lys-Lys-Lys (K3): (a) $[\text { Mal1P }+\mathrm{K} 3+2 \mathrm{H}]^{2+}$, (b) $[\mathrm{Lac} 1 \mathrm{P}+\mathrm{K} 3+2 \mathrm{H}]^{2+}$, (c) $[\text { Suc } 6 \mathrm{P}+\mathrm{K} 3+2 \mathrm{H}]^{2+}$. As expected, abundant ligand dissociation ions are observed in the CID data, which are characteristic for phosphorylated carbohydrates. These ions are marked with a star.

oxygen-sulfur bond cleavage. With an amine group present in the carbohydrate portion of the ion-pair complex, the complementary ions, singly charged peptide $[\mathrm{K} 3+\mathrm{H}]^{+}$and protonated disaccharide [IIIS + $\mathrm{H}]^{+}$, should be observed. The peptide ion is observed at $\mathrm{m} / \mathrm{z} 403.2$, but an ion corresponding to [IIIS $+\mathrm{H}^{+}$is not present in the spectrum. Since the peptide ion is observed, the dissociation reaction obviously occurs, but the ion corresponding to [IIIS $+\mathrm{H}]^{+}$probably dissociates by undergoing loss of $\mathrm{SO}_{3}$, producing the secondary ion at $m / z 417.8$.

In the MS/MS data of the ion pair complex [ $\Delta$ di- $6 \mathrm{~S}+$ $\mathrm{K} 3+2 \mathrm{H}]^{2+}$ in Figure $5 \mathrm{c}$, the observed characteristic ion is $m / z 380$, which originates from oxygen - sulfur bond cleavage. Also, the expected singly charged ions, protonated sulfated disaccharide $[\Delta \text { di- } 6 \mathrm{~S}+\mathrm{H}]^{+}(\mathrm{m} / \mathrm{z} 460)$ and the singly charged peptide ion $[\mathrm{K} 3+\mathrm{H}]^{+}(\mathrm{m} / z$ 403), are present. Thus, from these results it is apparent that the ion-pairing approach in combination with MS/MS experiments can be used to identify the phosphorylation and sulfation on doubly charged mono- and disaccharides. The characteristic product ions are useful in characterizing the acidic functional groups present on carbohydrates.
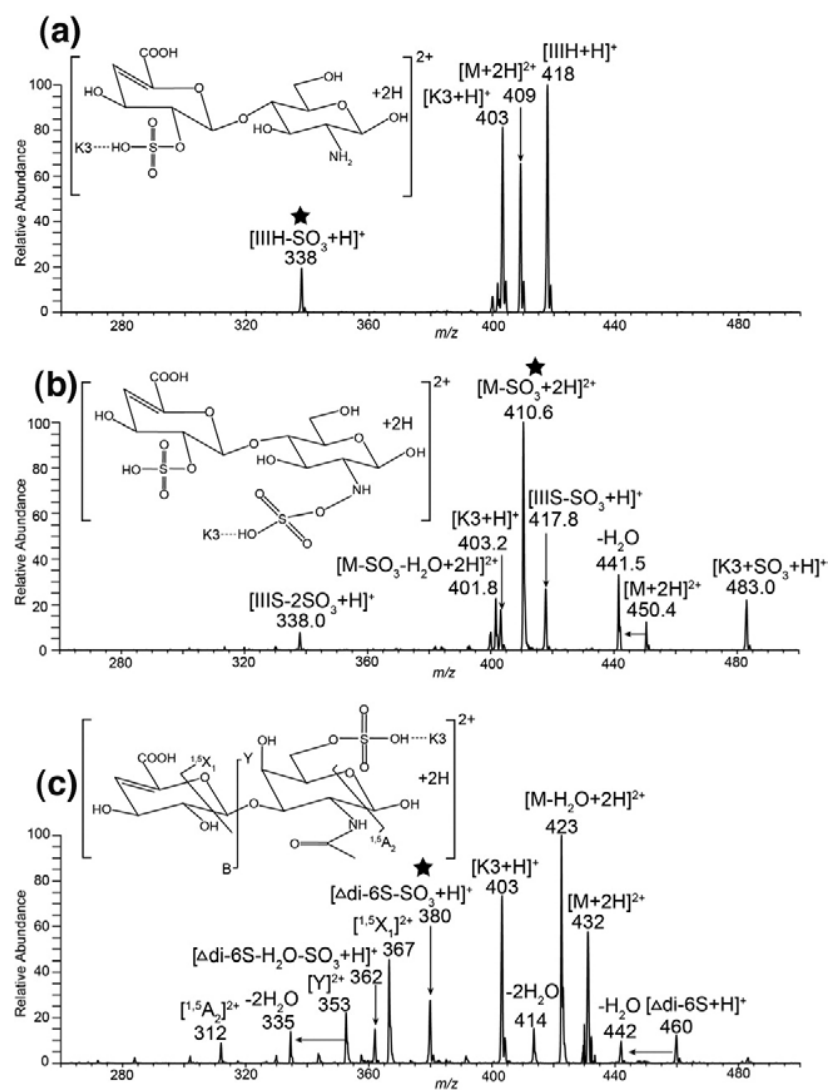

Figure 5. (+) ESI-MS/MS data for the doubly charged sulfated ion pairs with Lys-Lys-Lys (K3): (a) $[\mathrm{IIIH}+\mathrm{K} 3+2 \mathrm{H}]^{2+}$, (b) [IIIS $+\mathrm{K} 3+2 \mathrm{H}]^{2+}$, (c) $[\Delta \mathrm{di}-6 \mathrm{~S}+\mathrm{K} 3+2 \mathrm{H}]^{2+}$. As expected, the spectrum shows characteristic ions resulting from oxygen-sulfur bond cleavage. These ions are marked with a star.

\section{Biological Application}

To test the efficacy of using the ion-pairing approach in identifying functional groups on larger biomolecules, a highly sulfated glycoprotein hormone oLH $\alpha$ was used as a test case. In a previous study, the ion pairing approach was used to enhance the signal of mono-, di-sulfated glycopeptides from this protein [23]. Sulfated ion pairs can be readily observed in the ESI-MS analysis when adding the peptide $\mathrm{K} 3$ to the complex mixture [23]. This is because the peptide $\mathrm{K} 3$ suppresses the signal of neutral glycopeptides to facilitate the detection of sulfated glycoforms [23]. In this study, these glycopeptides are complexed with the peptide, $\mathrm{K} 3$, to demonstrate that the presence of sulfation on the structure can be confirmed by the ion-pairing method.

Four sulfated glycopeptides, which are present in the glycoprotein and contain different types of glycans, were selectively ion-paired with $\mathrm{K} 3$, and they formed abundant ion-pairing complexes at $\mathrm{m} / \mathrm{z}$ 1065.4, 1165.7, 1227.3, and 1146.3. The structures of the glycopeptides appear in Figure 6. To confirm that these glycopeptides contain sulfate and not phosphate, the ion-pairing complexes were subjected to CID experiments. MS/MS data of one of the precursor ions at $m / z 1065.4$ is shown in Figure 6a. The 

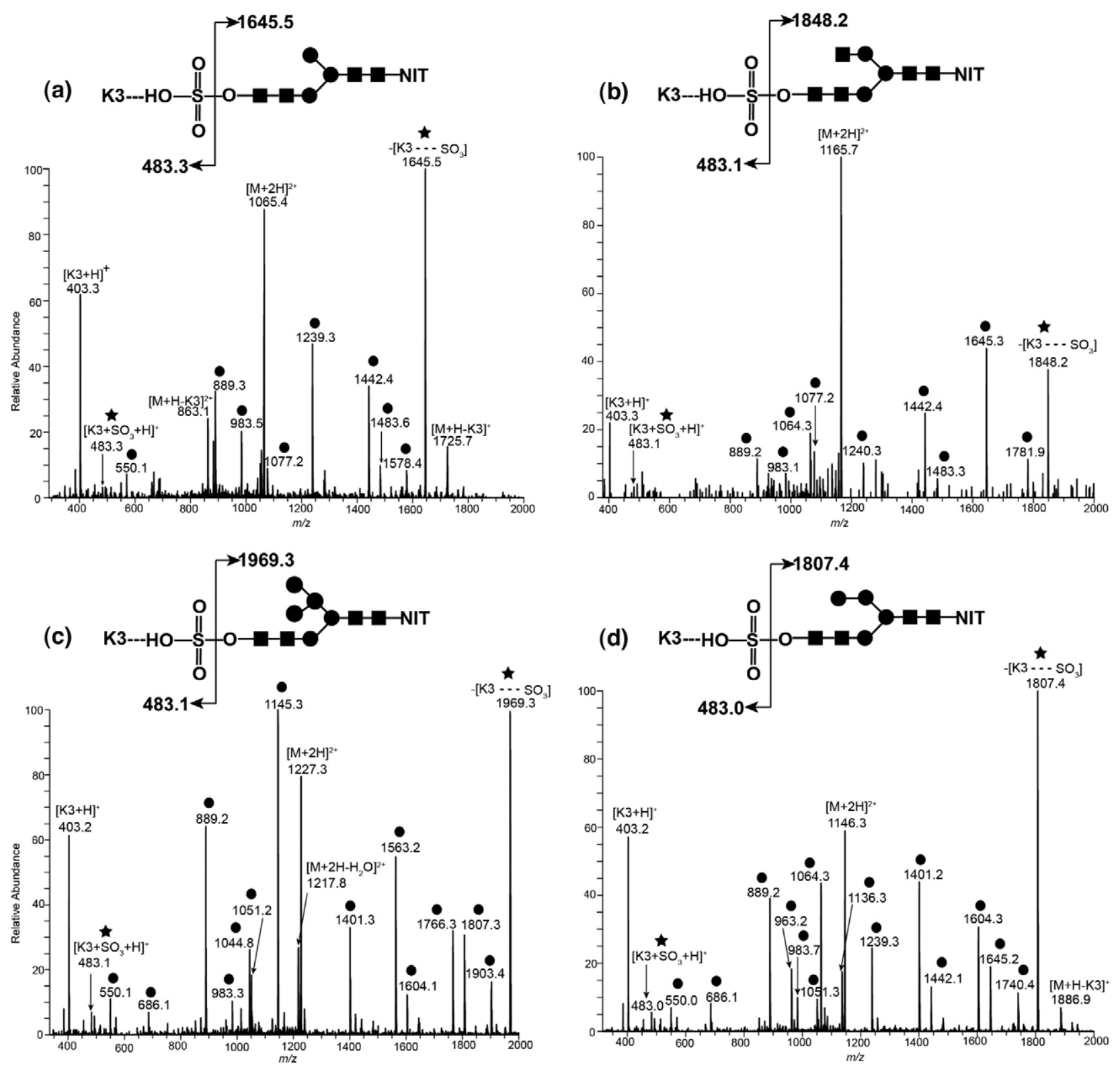

Figure 6. (+) ESI-MS/MS data for the doubly charged ion-pair complexes of glycopeptides from oLH $\alpha$ with Lys-Lys-Lys (K3): The characteristic ions marked with a star confirm that the glycopeptides are sulfated. The ions marked with a circle represent the ions that originated from glycosidic bond cleavage.

base peak $(m / z 1645.5)$ is an ion originating from oxygen - sulfur bond cleavage; this peak confirms that this glycopeptide is sulfated. Also, the ion at $\mathrm{m} / \mathrm{z} 483.3$ corresponding to $\left[\mathrm{K} 3+\mathrm{SO}_{3}+\mathrm{H}\right]^{+}$provides the additional evidence for the functional group's assignment. The complementary ions, singly charged K3 ( $\mathrm{m} / \mathrm{z} 403.3)$ and $[\mathrm{M}+\mathrm{H}-\mathrm{K} 3]^{+}(\mathrm{m} / \mathrm{z}$ 1725.73), are also expected. These ions correspond to dissociation of the noncovalent complex, and they are expected for sulfated HexNAc [22].

The other three glycopeptides (Figure 6b, c, and d) also dissociated to produce the product ions that correspond to oxygen - sulfur bond cleavage. These ions are at $\mathrm{m} / \mathrm{z}$ 1848.2 (Figure 6b), $m / z 1969.3$ (Figure 6c), and $m / z 1807.4$ (Figure 6d). This implies that all of these glycopeptides are sulfated, which is consistent with the results obtained from radiolabeling $[14,15]$. The ion at $m / z 403$, corresponding to the peptide ion $[\mathrm{K} 3+\mathrm{H}]^{+}$, is apparent on the spectrum of these glycopeptides. This ion indicates that the ion pairing complexes also undergo ligand dissociation, which is expected for sulfated HexNAc. Hence, the ion-pairing approach is useful to identify the modifications in complex biomolecules.

\section{Conclusions}

The differentiation between phosphorylation and sulfation on monosaccharides, disaccharides, and glycopeptides is accomplished using ion-pairing in combination 
with MS/MS experiments. The characteristic product ions of ion-pairing complexes are applicable in identifying the modification of larger biomolecules, such as glycopeptide mixtures obtained from ovine luteinizing hormone proteolytic digest. This is the first example of applying ion-pairing in discriminating the presence of phosphates and sulfates in complex biomolecules. This approach also has the added advantages of enhancing the signal of acidic functional groups in complex biological mixtures [23] as well as elucidating the structure of carbohydrate moiety of glycopeptides [24]. Therefore, this sulfate/phosphate discrimination strategy can be successfully incorporated into a single synergistic approach that can be used to structurally characterize phosphorylated or sulfated glycoproteins, without any separation or enrichment of the samples before analysis.

\section{Acknowledgments}

The authors acknowledge the NIH for funding (project number 1 P20 RR17708-01 and RO1GM077266), and Dr. George Bousfield of Wichita State University for supplying the glycopeptides.

\section{References}

1. Siegel, M. M.; Tabei, K.; Kagan, M. Z.; Vlahov, I. R.; Hileman, R. E.; Linhardt, R. J. Polysulfated carbohydrates analyzed as ion-paired complexes with basic peptides and proteins using electrospray negative ionization mass spectrometry. J. Mass Spectrom. 1997, 32, 760-772.

2. Mann, M.; Ong, S.; Gronborg, M.; Steen, H.; Jensen, O. N.; Pandey, A. Analysis of protein phosphorylation using mass spectrometry: Deciphering the phosphoproteome. Trends Biotechnol. 2002, 20, 261-268.

3. Rappsilber, J.; Steen, H.; Mann, M. Labile sulfo group allows differentiation of sulfotyrosine and phosphotyrosine in peptides. J. Mass Spectrom. 2001, 36, 832-833.

4. Hunter, T. The Croonian lecture 1997. The phosphorylation of proteins on tyrosine: Its role in cell growth and disease. Phil. Trans. R. Soc. Lond. B 1998, 353, 583-605.

5. Huttner, W. B. Protein tyrosine sulfation. Trends Biochem. Sci. 1987, 12, 361-363.

6. Delcommenne, M.; Kannagi, R.; Johnson, P. TNF- $\alpha$ increases the carbohydrate sulfation of CD44: Induction of 6-sulfo $\mathrm{N}$-acetyl lactosamine on $\mathrm{N}-$ and O-linked glycans. Glycobiology 2002, 12, 613-622.

7. Bai, X.; Brown, J. R.; Varki, A.; Esko, J. D. Enhanced 3-O-sulfation of galactose in Asn-linked glycans and Maackia amurensis lectin binding in a new Chinese hamster ovary cell line. Glycobiology 2001, 11, 621-632.

8. Xiong, L.; Andrews, D.; Regnier, F. Comparative proteomics of glycoproteins based on lectin selection and isotope coding. I. Proteome Res. 2003, 2, 618-625.
9. Sleat, D. E.; Sohar, I.; Pullarkat, P. S.; Lobel, P.; Pullarkat, R. K. Specific alterations in levels of mannose-6-phosphorylated glycoproteins in different neuronal ceroid lipofuscinoses. Biochem. J. 1998, 334, 547-551.

10. Lobel,, P., Karel,, R. Assay for glycoproteins containing mannose-6phosphate. PCT Int. Appl. 1997, 60.

11. Harvey, D. J. Structural determination of N-linked glycans by matrixassisted laser desorption/ionization and electrospray ionization mass spectrometry. Proteomics 2005, 5, 1774-1786.

12. Harvey, D. J. Fragmentation of negative ions from carbohydrates: Part 1. Use of nitrate and other anionic adducts for the production of negative ion electrospray spectra from N-linked carbohydrates. J. Am. Soc. Mass Spectrom. 2005, 16, 622-630.

13. Wheeler, S. F. Harvey, D. J. Extension of the in-gel release method for structural analysis of neutral and sialylated N-linked glycans to the analysis of sulfated glycans: Application to the glycans from bovine thyroid-stimulating hormone. Anal. Biochem. 2001, 296, 92-100.

14. Green, E. D.; Baenziger, J. U. Asparagine-linked oligosaccharides on lutropin, follitropin, and thyrotropin. I. Structure elucidation of the sulfated and sialylated oligosaccharides on bovine, ovine, and human pituitary glycoprotein hormones. J. Biol. Chem. 1988, 263, 25-35.

15. Green, E. D.; Baenziger, J. U. Asparagine-linked oligosaccharides on lutropin, follitropin, and thyrotropin. II. Distributions of sulfated and sialylated oligosaccharides on bovine, ovine, and human pituitary glycoprotein hormones. J. Biol. Chem. 1988, 263, 36-44.

16. Chen, S. L.; Huddleston, M. J.; Shou, W.; Deshaies, R. J.; Annan, R. S.; Carr, S. A. Mass spectrometry-based methods for phosphorylation site mapping of hyperphosphorylated proteins applied to Net 1, a regulator of exit from mitosis in yeast. Mol. Cell Proteomics 2002, 1, 186-196.

17. Annan, R. S.; Carr, S. A. Phosphopeptide analysis by matrix-assisted laser desorption time-of-flight mass spectrometry. Anal. Chem. 1996, 68, 3413-3421.

18. Oda, Y.; Nagasu, T.; Chait, B. T. Enrichment analysis of phosphorylated proteins as a tool for probing the phosphoproteome. Nat. Biotechnol. 2001, 19, 379-382.

19. Bossio, R. E.; Marshall, A. G. Baseline resolution of isobaric phosphorylated and sulfated peptides and nucleotides by electrospray ionization FTICR MS: Another step toward mass spectrometry-based proteomics. Anal. Chem. 2002, 74, 1647-1679.

20. Petzold, C. J.; Leavell, M. D.; Leary, J. A. Screening and identification of acidic carbohydrates in bovine colostrum by using ion/molecule reactions and Fourier transform ion cyclotron resonance mass spectrometry: Specific toward phosphorylated complexes. Anal. Chem. 2004, 76, 203210.

21. Harvey, D. J.; Bousfield, G. R. Differentiation between sulphated and phosphated carbohydrates in low-resolution matrix-assisted laser desorption/ionization mass spectra. Rapid Commun. Mass Spectrom. 2005, 19, 287-288.

22. Zhang, Y.; Go, E. P.; Jiang, H.; Desaire, H. A novel mass spectrometric method to distinguish isobaric monosaccharides that are phosphorylated or sulfated using ion-pairing reagents. J. Am. Soc. Mass Spectrom. 2005, 16, 1827-1839.

23. Jiang, H.; Irungu, J.; Desaire, H. Enhanced detection of sulfated glycosylation sites in glycoproteins. J. Am. Soc. Mass Spectrom. 2005, 16, 340-348.

24. Irungu, J.; Dalpathado, D. S.; Go, E. P.; Jiang, H.; Ha, H.; Bousfield, G. R.; Desaire, H. Method for characterizing sulfated glycoproteins in a glycosylation site-specific fashion, using ion pairing and tandem mass spectrometry. Anal. Chem. 2006, 78, 1181-1190.

25. Jiang, H.; Butnev, V. Y.; Bousfield, G. R.; Desaire, H. Glycoprotein profiling by electrospray mass spectrometry. J. Am. Soc. Mass Spectrom. 2004, 15, 750-758

26. Loo, J. A. Studying noncovalent protein complexes by electrospray ionization mass spectrometry. Mass Spectrom. Rev. 1997, 16, 1-23. 